C2018 IEEE. Personal use of this material is permitted. Permission from IEEE must be obtained for all other uses, in any current or future media, including reprinting/republishing this material for advertising or promotional purposes, creating new collective works, for resale or redistribution to servers or lists, or reuse of any copyrighted component of this work in other works. This is the author's version of an article that has been published in the conference proceedings. The final version of record is available at https://doi.org/10.1109/WCNC.2018.8377457 


\title{
Beyond GNSS: Highly Accurate Localization for Cooperative-Intelligent Transport Systems
}

\author{
S. Severi ${ }^{\dagger}$, H. Wymeersch ${ }^{\times}$, J. Härri ${ }^{\ddagger}$, M. Ulmschneider ${ }^{+}$, B. Denis* ${ }^{*}$ M. Bartels ${ }^{\diamond}$ \\ ${ }^{\dagger}$ Jacobs University Bremen, s.severi@jacobs-university.de ${ }^{\times}$Chalmers University of Technology, henk@chalmers.se \\ ${ }^{\ddagger}$ EURECOM, jerome.haerri@eurecom.fr ${ }^{+}$German Aerospace Center (DLR), markus.ulmschneider@dlr.de \\ *CEA-Leti, benoit.denis@cea.fr ${ }^{\diamond}$ Ibeo Automotive Systems GmbH, marcus.bartels@ibeo-as.com
}

\begin{abstract}
This positioning paper provides an overview on an envisioned platform, intended as a set of technologies, protocols and algorithms, to achieve highly accurate localization for cooperative-intelligent transport systems. This is the result of a three years investigation conducted within the scope of the EU H2020 HIGHTS project and offers an insight on the envisioned hybrid service platform to enable vehicular positioning services for highly automated driving (HAD) scenarios. This paper reviews the main components of such a platform, drafting the guidelines for the seamless integration (i.e. hybridization) and field validation of multiple localization solutions to support robust HAD functionalities.
\end{abstract}

\section{INTRODUCTION}

Autonomous driving is certainly the hype of the moment within the Intelligent Transport System (ITS) sector. Although we are relatively far from a futuristic vision where all vehicles on the road are fully autonomous, day one technologies for Highly Automated Driving (HAD) are expected to bring a huge improvement for the safety and protection of Vulnerable Road Users (VRUs). A core capability of every autonomous vehicle is therefore the geo-temporal awareness, i.e., to know, with significant confidence, both its own real-time position and those of all other entities (e.g., surrounding vehicles, VRUs, road boundaries and lanes, road artifacts and objects), i.e., the so-called electronic horizon. Such awareness is based on a vehicular localization system that goes beyond GNSS in terms of both accuracy and continuity.

The EU H2020 HIGHTS project has been putting forward the fundamental idea of a cooperative hybrid service platform, collecting geo-temporal inputs from different technologies to enable new vehicular positioning services that require accurate and resilient Local Dynamic Maps (LDMs). The design, the implementation and the proofof-concept validation of this platform are key technical outcomes of the HIGHTS project. One central aspect of the envisioned service platform is the cooperation between vehicles for sensing and positioning. In order words, carcentric sensor data transmitted between vehicles will be even more beneficial if their context is also shared between cars. This is the purpose of LDMs, which will be available on future ITS. Maps are central to any positioning solution, as they assist cooperative positioning algorithms with potentially known landmarks, Global Navigation Satellite System (GNSS) for map matching, and more generally
TABLE I

LOCALIZATION TECHNOLOGIES

\begin{tabular}{|c|c|c|}
\hline Technology & Benefits & Drawbacks \\
\hline GNSS & $\begin{array}{l}1.5 \text { meters absolute posi- } \\
\text { tioning accuracy }(20 \mathrm{~cm} \\
\text { for RTK) }\end{array}$ & $\begin{array}{l}\text { needs Line-of-Sight } \\
\text { (LOS) between satellites } \\
\text { and receiver }\end{array}$ \\
\hline Lidar & $\begin{array}{l}\text { cm-level accuracy } \\
\text { tracking and identification } \\
\text { of objects }\end{array}$ & $\begin{array}{l}\text { needs line of sight, re- } \\
\text { quires map for absolute } \\
\text { localization }\end{array}$ \\
\hline Radar & $\begin{array}{l}\text { cm-level accuracy, track- } \\
\text { ing and identification of } \\
\text { objects }\end{array}$ & less accurate than Lidar \\
\hline Odometer & simple, low cost & only measures distance \\
\hline Camera & $\begin{array}{l}\text { recognition of environ- } \\
\text { mental objects }\end{array}$ & $\begin{array}{l}\text { only relative information, } \\
\text { needs line of sight }\end{array}$ \\
\hline $\mathrm{V} 2 \mathrm{X}$ & $\begin{array}{l}\text { sharing of measurement } \\
\text { data } \\
\text { extract range information } \\
\text { from radio signals }\end{array}$ & $\begin{array}{l}\text { unreliable wireless chan- } \\
\text { nel } \\
\text { poor accuracy of measure- } \\
\text { ments }\end{array}$ \\
\hline
\end{tabular}

provide an enhanced environmental perception for future autonomous vehicles. Despite the fact that maps represent a fundamental part of positioning systems, multiple challenges related to their uniform usage arise, such as differences in map technology and data structures. Hence, it is necessary to define recommendations and promote standardization of ontologies and semantics for roads, objects, communication channel properties, navigation and any other similar aspect. The ETSI ITS integrated a specification for LDMs [1], [2], which should be extended to integrate the additional sensor and contextual data from HAD and VRU services. Another challenge is to make this enhanced LDM specification compact and efficient, with data classified in layers. Finally, due to the required delay to guarantee map freshness and the potential large capacity required to transfer mapping data, the location of servers needs to be well planned, possibly with preference of edge-based solutions over cloud ones. Despite the multiple and heterogeneous positioning technologies already present in the market (see Table I), both in term of GNSS-based systems and on-board sensors, none of them are effective under all road conditions and scenarios. However, suitable fusion into a hybrid architecture will allow to overcome all the peculiar limitations enabling a robust, resilient and effective service platform.

In this paper, we describe the main concepts of this cooperative hybrid service platform, architecture implementation, and early field validation. 


\section{Communication Technologies and Protocols}

Besides accuracy requirements, the envisioned hybrid service platform also aims at a latency level compatible with Cooperative Intelligent Transport System (C-ITS) applications. The approach is to rely on disruptive cooperative V2X communication strategies and protocols (i.e., between vehicles, infrastructure elements and possibly, VRUs and/or Internet-of-Things (IoT) devices) to exchange and consolidate positioning and contextual information, thus complementing the data produced by on-board systems. The aim is to exchange data of various kind and quality (ranging, GNSS data, maps, caching, contexts), which can be used by the cooperative fusion algorithms described in Section III.

A second objective is to develop methodologies to enrich the LDM with high precision maps, local elementary views of vehicles and dangers, environmental landmarks, or statistical data (space-time mobility/connectivity contexts).

The main challenges of the communication subsystem are thus threefold:

- Communication Capacity - any available communication technology shall be used to support the required data volume and delay between ITS stations and services.

- Message Format - none of the four types of messages within the current C-ITS architecture has been specified to support the exchange of environmental/contextual data.

- Contextual Information - most of the information gathered by one ITS station will be meaningful in a specific context, which also needs to be exchanged in an understandable way.

To this end, six current state-of-the-art communication technologies have been used for testing within HIGHTS projects (ETSI ITS-G5 V2X, Bluetooth, IEEE 802.15.4, ZigBee, WiFi-Direct and 4g LTE) and two other technologies (3GPP LTE-D2D and mm-WAVE) have been considered for more prospective investigations and future developments. Table II summarizes their main characteristics.

\section{Hybrid Localization with Heterogeneous INFORMATION}

This chapter offers an overview on the main algorithmic solutions envisioned within the HIGHTS project to perform hybrid vehicular localization, i.e. to do positioning with heterogeneous information.

\section{A. Implicit Cooperative Positioning}

Explicit range measurements between vehicles can be performed with high accuracy when using dedicated technologies, including UWB or the Fine-Timing-Measurement (FTM) protocol defined in IEEE $802.11 \mathrm{mc}$. When such technologies are unavailable, $\mathrm{V} 2 \mathrm{~V}$ and V2I radio links can still be used for implicit cooperative positioning (ICP). The aim of ICP is to improve the localization accuracy of vehicles by having multiple vehicles observe the same scene and sharing information about this scene. In ICP, at time step $k$, each vehicle $i$ has a local GNSS measurement of its own state $y_{i, k}$ in a global coordinate system, and a local measurement $z_{i, k}$ of surrounding mobile objects (vehicles, pedestrians, or other objects not available in offline maps) in the scene, obtained from an on-board sensor, such as a radar, LIDAR, or stereo camera. Denoting by $x_{i, k}$ the state of vehicle $i$ (position, heading, velocity) and by $s_{k}$ the state of the objects (position, velocity), measurements are of the form

$$
\begin{aligned}
& y_{i, k}=f\left(x_{i, k}\right)+n_{i, k} \\
& z_{i, k}=g\left(x_{i, k}, s_{k}\right)+w_{i, k},
\end{aligned}
$$

where $f(\cdot)$ is the GNSS measurement function, $g(\cdot, \cdot)$ is the vehicle-to-object measurement function, which depends both on the state of the vehicle and the state of the mobile objects, and $n_{i, k}$ and $w_{i, k}$ are stochastic measurement errors. Aggregating all measurements in $\mathbf{y}_{k}$ and $\mathbf{z}_{k}$, the goal of ICP is to determine $p\left(x_{i, k} \mid \mathbf{y}_{k}, \mathbf{z}_{k}\right)$ through either centralized (with V2I) or distributed processing (with $\mathrm{V} 2 \mathrm{~V}$ ). This problem is challenging as vehicles may not be able to perceive the complete $s_{k}$ and $g(\cdot, \cdot)$ may be invariant to permutations of the objects (as objects have no natural identifier). The ICP determines approximations of $p\left(x_{i, k} \mid \mathbf{y}_{k}, \mathbf{z}_{k}\right)$ through Bayesian filtering, as described in [3], [4].

\section{B. Cholesky MDS}

Consider a vehicular network of $N$ nodes in a 2dimensional Euclidean space. There are $N_{a}$ nodes with known location (anchors) and $N_{t}$ nodes with unknown location (targets) which are to be localized. Such a network can be represented by an undirected graph $G_{\eta, N}(\mathbf{Z}, \mathrm{V}, \mathrm{D})$, with vertices $\mathbf{Z}=\left\{\mathbf{z}_{1}, \ldots, \mathbf{z}_{N_{a}}, \mathbf{z}_{N_{a}+1}, \ldots, \mathbf{z}_{N_{a}+N_{t}}\right\}$, undirected edges $\mathrm{V}=\left\{v_{n, m}\right\}$ and weights $\mathrm{D}=\left\{d_{n, m}\right\}$, where $n, m \in\{1, \cdots, N\}$.

Here edges represent communication links while weights are the Euclidean distances between sources, given by

$$
d_{n, m}=\mathcal{D}\left(\mathbf{z}_{n}, \mathbf{z}_{m}\right) \triangleq \sqrt{\left\langle\left(\mathbf{z}_{n}-\mathbf{z}_{m}\right) ;\left(\mathbf{z}_{n}-\mathbf{z}_{m}\right)\right\rangle},
$$

where $\left\langle\mathbf{z}_{n} ; \mathbf{z}_{m}\right\rangle$ denotes the inner product of $\mathbf{z}_{n}$ and $\mathbf{z}_{m}$.

The new proposed algorithm, namely Cholesky MDS (CMDS), creates the so called Edge-Gramian Kernel (EGK) $\mathbf{K}$ based on the distance and (optionally) angle information to estimate, via algebraic manipulations (full details are provided in [10]) to finally derive $\mathbf{Z}$.

More in details, the EGK can be written as product of a lower and upper triangular matrix

$$
\begin{aligned}
\mathbf{K} & =\mathbf{V} \cdot \mathbf{V}^{\dagger} \\
& =\left[\begin{array}{cccc}
v_{1} & 0 & \cdots & 0 \\
v_{N} & v_{2} & \cdots & 0 \\
\vdots & \vdots & \ddots & \vdots \\
v_{M} & v_{M-1} & \cdots & v_{N-1}
\end{array}\right] \cdot\left[\begin{array}{cccc}
v_{1}^{*} & v_{N}^{*} & \cdots & v_{M}^{*} \\
0 & v_{2}^{*} & \ddots & v_{M-1}^{*} \\
\vdots & \vdots & \ddots & \vdots \\
0 & 0 & \cdots & v_{N-1}^{*}
\end{array}\right],
\end{aligned}
$$

where ${ }^{\dagger}$ is the conjugate transpose operator and $\mathbf{V}$ contains all the individual edges represented in complex form such that

$$
v_{i}=\left(z_{m, x}-z_{n, x}\right)+j\left(z_{m, y}-z_{n, y}\right) .
$$


TABLE II

COMMUNiCATION CAPABILITIES B Y CURRENT AND NEW TECHNOLOGIES [7]

\begin{tabular}{|c|c|c|c|c|c|c|}
\hline Technologies & Throughput & Delay & D2D & Range & Sec/Privacy & Ranging Cap. \\
\hline ITS-G5 & 3-12Mbps & $\sim 10 \mathrm{~ms}$ & Yes & $300-1000 \mathrm{~m}$ & Strong & No \\
\hline Bluetooth & $24 \mathrm{Mbps}$ & $\sim 100 \mathrm{~ms} \#$ link & Yes & $20-30 \mathrm{~m}$ & Weak & No \\
\hline IEEE 802.15.4 & $250 \mathrm{Kbps}$ & 〜20ms\#link & Yes & $10 \mathrm{~m}$ & Weak & Yes \\
\hline UWB & $25 \mathrm{Gbps}$ & $\sim 20 \mathrm{~ms} \#$ link & Yes & $10 \mathrm{~m}$ & Weak & Yes \\
\hline 3GPP LTE & 100Mbps UL/50 Mbps DL & $\sim 250-100 \mathrm{~ms}$ & No & $>1 \mathrm{Km}$ & Strong & No \\
\hline WiFi (Direct) & $1 \mathrm{Gbps}$ & $\sim 1 \mathrm{~s}$ & Yes & $\sim 100 \mathrm{~m}$ & Weak & No \\
\hline \multicolumn{7}{|c|}{ New Technologies } \\
\hline LTE D2D & $\sim 70 \mathrm{Mbps}$ & $\sim 50 \mathrm{~ms}$ Mode1/ 10ms Mode2 & Yes & $300-900 \mathrm{~m}$ & Mode 1 Yes/Mode 2 No & Not defined \\
\hline mmWAVE & $>10 \mathrm{Gbps}$ & $1 \mathrm{~ms}$ e2e & Yes & $<200 \mathrm{~m}$ & Implicit & Not defined \\
\hline
\end{tabular}

The Cholesky algorithm can be briefly described by the following pseudo-code:

\begin{tabular}{l}
\hline Algorithm $\mathbf{1}$ Cholesky MDS \\
\hline Input: \\
- Pairwise distance and angle estimates. \\
- The coordinates of at least $N_{a} \geq\left\lceil\frac{1+\sqrt{8 N-7}}{2}\right\rceil$. \\
Steps: \\
1. Construct the kernel $\mathbf{K}$. \\
2. Compute $\hat{\mathbf{V}}$ via Cholesky-decomposition of $\mathbf{K}$. \\
3. Compute $\hat{\mathbf{Z}}$. \\
4. Retrieve $\mathbf{Z}$ from $\hat{\mathbf{Z}}$ via Procrustes transformation. \\
\hline
\end{tabular}

\section{Virtual Anchor Cooperative Localization}

In Vehicular Ad hoc NETworks (VANETs), an "ego" vehicle can consider its neighbors as potential "virtual anchors" [12], [13], [11] (i.e. mobile anchors with only approximate knowledge about their own positions). The principle of our vehicular Virtual Anchors assisted Cooperative Localization (VA-CLoc) works in three phases. First, each vehicle piggybacks its absolute position information in a "Beacon" sent over "V2X" communication links ${ }^{1}$. Through the reception of these "Beacons", a given "ego" vehicle becomes aware of the absolute position estimates of its neighbors. The second phase consists of using the "Beacon" signal statistics to sample relative position-dependent information from these "virtual anchors" (e.g., Vehicleto-Vehicle (V2V) distances, relative angles, etc.). Ad hoc trilateration can then be locally applied to fuse the latter information with on-board GNSS position estimates and further enhance the absolute localization (see Fig. 1). In the final phase, the "ego" vehicle cooperates to improve the localization of other vehicles by further broadcasting its fusion results in subsequent "Beacons". CLoc has already been applied in [12], [13], [11] to fuse on-board GPS positions with opportunistic V2V Received Signal Strength Indicators (RSSIs) out of "Beacons" such as Cooperative Awareness Messages (CAMs), relying on the V2X ITS-G5 technology. ${ }^{2}$ A major advantage of using V2V RSSI lies in

\footnotetext{
${ }^{1}$ To remain technology neutral, a "Beacon" is a message periodically broadcast by each node, while "V2X" (Vehicle-to-X) refers to any technology capable of Device-to-Device (D2D) communication in a vehicular context.

${ }^{2}$ CAM and ITS-G5 are European counterparts to the Basic Safety Message (BSM) and Dedicated Short Range Communication (DSRC) in the US.
}

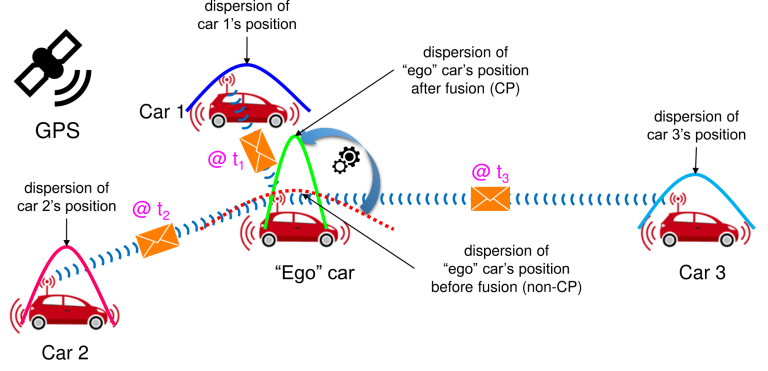

Fig. 1. "Ego" car receiving asynchronous CAMs from one-hop "virtual anchors" to perform distributed CLoc. The dispersion of CLoc location estimates (through both GNSS and ITS-G5) is expected to be lower than that of non-CLoc estimates (i.e., standalone GPS).

the full compliance with future ITS-G5 connected vehicles.

\section{Channel-SLAM}

The fundamental idea of Channel-SLAM is to exploit the information contained in the multipath components of signals of opportunity. In particular, each multipath component arriving at a receiver via a different propagation path is regarded as a signal from a so-called virtual transmitter in a pure LOS condition to the receiver. While the derivation and a detailed description are presented in [8], Figure 2 illustrates the idea of Channel-SLAM, with one physical transmitter $\mathrm{Tx}$ in the scenario. The signal component in blue reflected at the surface and arriving at the user is treated as a LOS signal from the virtual transmitter $\mathrm{vTx}_{1}$. Geometrical considerations show that the location of $\mathrm{vTx}_{1}$ is the location of Tx mirrored at the reflecting surface, and that the two transmitters are inherently perfectly synchronized. The signal component in green scattered at the punctual scatterer is regarded as being transmitted by the virtual transmitter $\mathrm{vTx}_{2}$, which is at the scatterer location. Though, in the case of scattering, there is a delay offset $\tau_{0}$ among the physical and the virtual transmitter corresponding to the Euclidean distance between the two. The delay offset can be regarded as a clock offset. In both cases, the locations and delay offsets of the virtual transmitters are static as the user is moving.

Channel-SLAM works in two stages. In the first stage, the Time of Arrival (ToA) and Angle of Arrival (AoA) of each multipath component are estimated. No knowledge on the geometry of the scenario is assumed, and hence the locations of the physical and virtual transmitters are unknown. The 


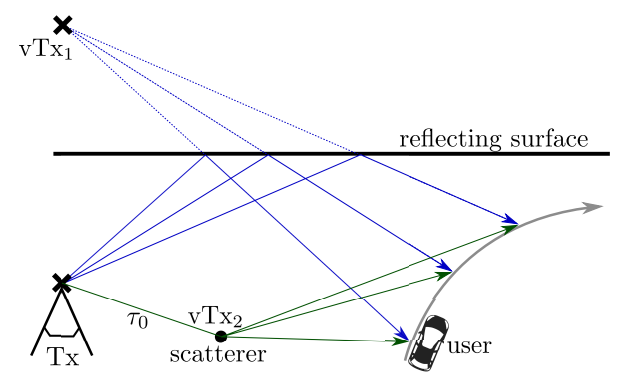

Fig. 2. A physical transmitter Tx broadcasts a signal. The blue multipath component arriving at the user via the reflecting surface is treated as a LOS signal from the virtual transmitter $\mathrm{vTx}_{1}$ and the green multipath component scattered at the punctual scatterer as a LOS signal from the virtual transmitter vTx 2 . The location of both virtual transmitters is static as the user moves.

estimates from the first stage are used as measurements in the second stage to simultaneously localize the user and map the physical and virtual transmitters with a Rao-Blackwellized particle filter in a Simultaneous Localization and Mapping (SLAM) approach. The user state consists of the position and velocity in two dimensions each. For each transmitter, its location and clock offset are estimated. In particular, we assume that different virtual transmitters correspond to multipath components interacting with distinct objects, and hence the transmitters are estimated separately.

As a user moves through a scenario, different physical and virtual transmitters can be observed for a certain time. When a new transmitter is detected, it is important to know if this transmitter had been observed before, and if so, to which of the previously observed transmitters the new one corresponds. This problem is known as data association. Data association is crucial for long-term robust SLAM, and hence, Channel-SLAM has been expanded by an association method in [9].

While users moves through a scenario, they estimate the states of physical and virtual transmitters, and thus create maps of transmitters. Such transmitter maps can be shared among users as features of LDMs in an ITS context.

\section{ARCHITECTURE AND IMPLEMENTATION APPROACH}

An on-board demonstrator setup for experimental validation of the cooperative localization approaches presented here is realized as part of HIGHTS. Figure 3 shows the generic architecture of this realization approach. The sensing sub-systems provide measurements from different sensing technologies, while the communication sub-system exchanges data with other vehicles and infrastructure. Table III gives an overview of the technologies integrated in HIGHTS. Further main elements are the LDM, the data fusion plane hosting the hybrid localization algorithms (described in Section III), as well as the interfaces between these modules.

At each vehicle, the LDM is realized by means of notebook PC hardware hosting a MongoDB database to collect, store and provide the sensor data as well as the data fusion

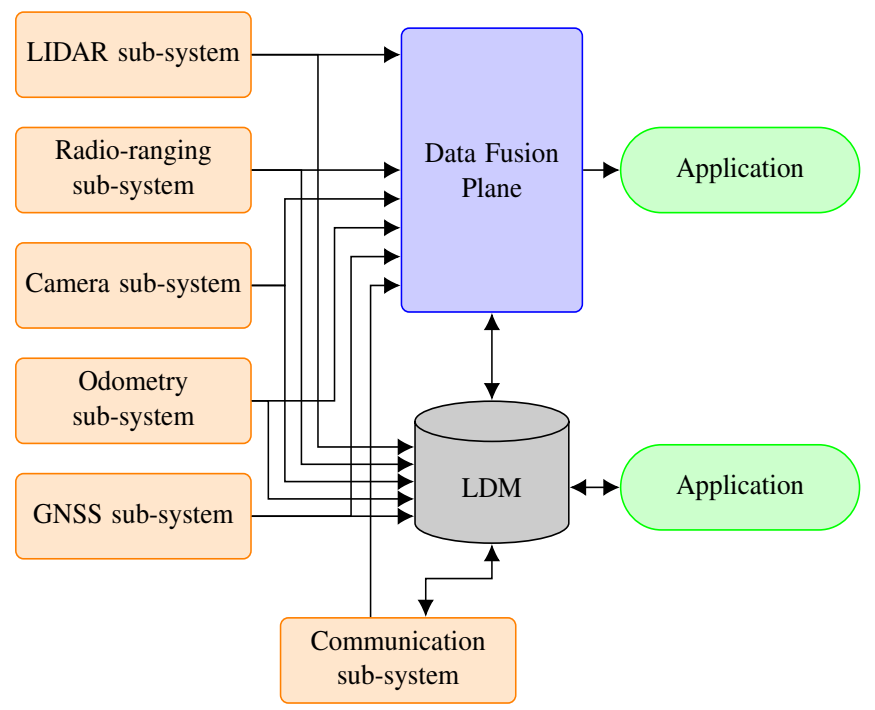

Fig. 3. Generic system architecture of the demonstrator setup.

TABLE III

DATA PROVIDED BY THE SUB-SYSTEMS

\begin{tabular}{|l|l|l|}
\hline Sub-system & Technology & Provided data \\
\hline \hline Lidar & $\begin{array}{l}\text { object tracking based on the } \\
\text { history of scan echoes }\end{array}$ & $\begin{array}{l}\text { object ID, relative } \\
\text { object position }\end{array}$ \\
\hline $\begin{array}{l}\text { V2X Radio- } \\
\text { ranging }\end{array}$ & $\begin{array}{l}\text { IEEE 802.15.4 phase mea- } \\
\text { surement, UWB time-of-flight } \\
\text { measurement, RSSI-based dis- } \\
\text { tance estimation for ITS-G5 }\end{array}$ & station ID, range \\
\hline Camera & Lane boundary detection & $\begin{array}{l}\text { distance to lane } \\
\text { marking }\end{array}$ \\
\hline Odometry & $\begin{array}{l}\text { Ego-motion estimation based } \\
\text { on IMU and wheel-speed sen- } \\
\text { sors }\end{array}$ & $\begin{array}{l}\text { velocity, heading, } \\
\text { yaw rate }\end{array}$ \\
\hline GNSS & $\begin{array}{l}\text { Satellite-based global position- } \\
\text { ing via GPS }\end{array}$ & latitude, longitude \\
\hline Communication & $\begin{array}{l}\text { ITS-G5 V2X Communication } \\
\text { (Cohda MK5) }\end{array}$ & $\begin{array}{l}\text { neighbor ID, posi- } \\
\text { tion and velocity }\end{array}$ \\
\hline
\end{tabular}

results. The LDM is connected to all sub-systems via onboard Ethernet or CAN bus (via CAN gateway). All data are time-referenced, a common time base is ensured by using Network Time Protocol (NTP) synchronization based on GPS. The database is organized in groups by means of the sub-systems, following the W3C Automotive naming convention. A critical requirement to the platforms used for implementation of the algorithms is the ability to execute the required computations in real time.

\section{VALIDATIONS}

\section{A. Experimental Settings}

Preliminary offline validations of the algorithms ${ }^{3}$ described in Sec. III have been conducted based on experimental data.

\footnotetext{
${ }^{3}$ Channel-SLAM could not be validated, given the available technologies integrated onto the proof of concept platform by the time of these trials. The reader is invited to refer to [6] for a more complete simulation-based performance assessment.
} 
a) Scenario 1: One first large-scale trial took place at TASS' vehicular test facilities in Helmond, Netherlands. The involved platoon consisted of 3 equipped cars driving in line (See Fig. 4). Each vehicle was equipped according to Sec. IV, except for the data fusion plane, which was not yet realized at this stage. The vehicles made two full rounds along the A270/N270 highway section. The followed route deliberately included a combination of straight and curvy portions of road for better representativity and more realistic performance assessment ${ }^{4}$. Ground-Truth (GT) positions were logged using the on-board Real Time Kinematic (RTK) GPS.

While evaluating ICP, the first leading vehicle was viewed as the "Ego" vehicle under test, using uniquely its lowaccuracy GPS for fusion, whereas the high-accuracy RTK GPS of the second vehicle was used to assist and improve the former. Three well-identified features detected by the onboard lidars of both vehicles (uniformly distributed along the road and separated by approximately $30 \mathrm{~m}$ ) were extracted as observations. Performance was evaluated over a short time period when at least one of these features was simultaneously detected by the two lidars (i.e., for about $7 \mathrm{sec}$ ) in a harsh area for standard GPS. The fusion result was updated every $100 \mathrm{~ms}$ to benefit from lidar refreshment rate, after manually removing biases (measurement artifacts), without loss of generality about the findings accounted hereafter.

As for VA-CLoc, the third and last vehicle of the platoon was considered as the "Ego" one, receiving CAMs encapsulating RTK GPS data from both the first and second vehicles, measuring their respective RSSIs as explicit $\mathrm{V} 2 \mathrm{~V}$ range-dependent measurements, and finally performing fusion with its own on-board GPS data. Relying on mobilitybased prediction, the "Ego" vehicle also re-synchronized the RTK GPS information received from its neighbors, up to the fusion time. The CAMs rate was approximately $3 \mathrm{~Hz}$, whereas the fusion rate was set to 8 or $4 \mathrm{~Hz}$. Finally, so as to calibrate the required $\mathrm{V} 2 \mathrm{~V}$ path loss model parameters, we considered the RSSI readings collected over all the trials and all pairs of MK5 devices, along with the GT distance based on the three embedded RTK receivers. The fitted path loss exponent (2.5) and shadowing standard deviation (3.7 $\mathrm{dB}$ ) were further used in the observation model of the fusion filter.

b) Scenario 2: One more focused test took place at Zigpos headquarters, in a parking lot equipped with 6 static anchor nodes (see Fig. 5). This scenario was primarily intended to evaluate the suitability of CMDS at moderate speed in GNSS-denied urban environments. This algorithm is indeed viewed as a fast low-complexity alternative to GNSS, for instance for initializing other fusion algorithms (e.g., VA-CLoc, ICP...), while relying on ranging-enabled Road Side Units (RSUs) or static parked cars serving as fixed anchors. Input V2I range measurements were collected based on the Impulse Radio-Ultra Wideband (IR-UWB)

\footnotetext{
${ }^{4} \mathrm{An}$ interactive map is provided at http://u.osmfr.org/m/151124
}

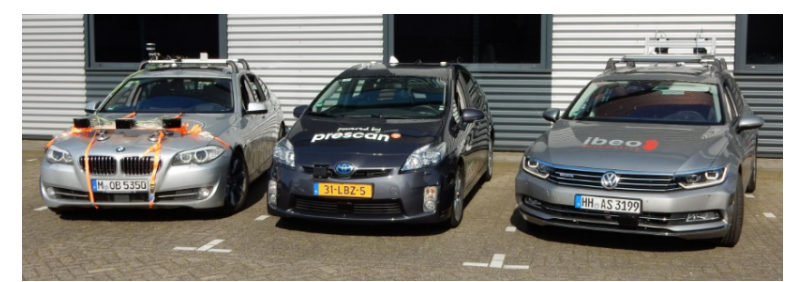

Fig. 4. Test vehicles involved in the first HIGHTS field trials carried out in Helmond: Objective's BMW, TASS's Prius and Ibeo's Passat (left to right).

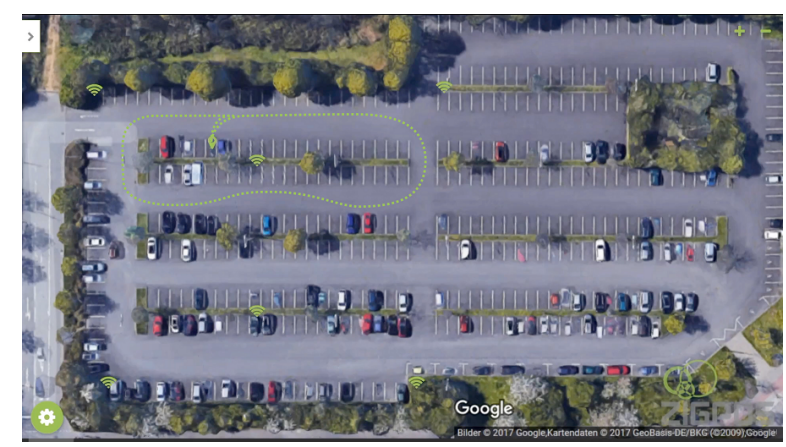

Fig. 5. Test site and estimated vehicle's trajectory (green) on a parking lot in Dresden, Germany - Sc. 2 (original pic. from Google Map).

technology embedded inside the radiolocation sub-system of the proof of concept platform.

\section{B. Results and Discussions}

Table IV summarizes the 2D location errors achieved with ICP and VA-CLoc in the first scenario for three characteristic values of their empirical cumulative density function (ECDF) (i.e., 0.1, 0.5 and 0.9 respectively).

Despite a relatively low fusion rate of $4 \mathrm{~Hz}$, VA-CLoc is shown to outperform standard GPS on both trips, even though the gain is somehow lower than expected (provided that neighbors are endowed with accurate RTK GPS and thus, could have been more helpful). This is partly due to the large dispersion of V2V RSSI measurements, but also to the low number of cooperative neighbors involved in the test case (only 2, at most), to the relatively low CAM rate while providing RSSIs and neighboring positions (at approximately $3 \mathrm{~Hz}$ in average, compared to the expected $10 \mathrm{~Hz}$ nominal rate) and finally, to unfavorable geometric dilution of precision (GDoP) conditions (as the three vehicles were forming a longitudinal platoon most of the time and the "Ego" vehicle was peripheral). Regarding the latter issue, further investigations have confirmed that the 2D location error is by far dominated by its cross-track component, whereas the cooperation gain is mostly visible on the alongtrack dimension. Other off-line tests have also shown that increasing the fusion rate up to $8 \mathrm{~Hz}$ in this case is however not really beneficial to $\mathrm{V} 2 \mathrm{~V}$ cooperation in the sense most of the iterations just correspond to filtered GPS but not to a true fusion event and thus, the amount of information remains approximately the same. For next field trials, we thus recommend higher V2x IST-G5 transmission rates (up 
TABLE IV

2D Location ERrors IN SCENARIO 1 AT 'Ego" VEHICLE

\begin{tabular}{|l|l|l|l|}
\hline Critical ECDF values & $10 \%$ & $50 \%$ & $90 \%$ \\
\hline $\begin{array}{l}\text { Raw GPS (local/harsh GPS } \\
\text { area, 1st veh.) - 1st trip (7sec) }\end{array}$ & $6.8 \mathrm{~m}$ & $6.9 \mathrm{~m}$ & $7.1 \mathrm{~m}$ \\
\hline $\begin{array}{l}\text { ICP (local/harsh GPS area, } \\
\text { 1st veh.) - 1st trip (7sec) }\end{array}$ & $3.5 \mathrm{~m}$ & $3.8 \mathrm{~m}$ & $4.2 \mathrm{~m}$ \\
\hline $\begin{array}{l}\text { Raw GPS (full trajectory, 3rd } \\
\text { veh.) - 1st/2nd trips }\end{array}$ & $2.5 \mathrm{~m} / 2.1 \mathrm{~m}$ & $3.6 \mathrm{~m} / 3.0 \mathrm{~m}$ & $>5 \mathrm{~m} / 4.7 \mathrm{~m}$ \\
\hline $\begin{array}{l}\text { VA-CLoc (full trajectory, 3rd } \\
\text { veh.) - 1st/2nd trips }\end{array}$ & $0.6 \mathrm{~m} / 0.2 \mathrm{~m}$ & $2.1 \mathrm{~m} / 1.6 \mathrm{~m}$ & $4.0 \mathrm{~m} / 3.2 \mathrm{~m}$ \\
\hline
\end{tabular}

to $10 \mathrm{~Hz}$ ), the use of additional V2I RSSI measurements with respect to ITS-G5 enabled RSUs (serving as anchors), a more realistic platoon topology varying over time, as well as the use of complementary ranging-enabled technologies such as IR-UWB (e.g., for V2V measurements).

Regarding ICP, despite an even more modest cooperation level (i.e., 1 side vehicle only) and a quite restrictive sensing scenario (i.e., evaluation over $7 \mathrm{sec}$ with 3 sensed features at most), significant gains larger than $40 \%$ in terms of both the median and worst-case positioning errors could already be noticed in comparison with nominal GPS performance (e.g., from approximately $7 \mathrm{~m}$ down to $4 \mathrm{~m}$ ). The gain looks even slightly better in the lower error regime. However, several critical issues such as precise GPS/LIDAR synchronization, distributed association procedures on specific (static) sensed features, or translation errors in feature detections (presumably propagated vehicle pose errors, were manually corrected here), will have to be addressed for next field validations.

As for Scenario 2, RTK data were not yet available at the vehicle under test for GT comparisons. Accordingly, only qualitative localization performance could be assessed for CMDS. However, the implemented algorithm was shown to be functional and could deliver consistent outputs in compliance with the accuracy level required in the considered scenario [14], that is to say, approximately on the same order of the $30 \mathrm{~cm} \mathrm{V2X} \mathrm{IR-UWB} \mathrm{ranging} \mathrm{accuracy} \mathrm{(as}$ characterized in [6]). Other complementary tests conducted in the first scenario (though not reported herein) have pointed out the current limitations of the Zigbee-based ranging technology at high speed and/or in case of adverse deployment of fixed anchors along the road (in terms of both spatial coverage and geometric dilution of precision). Accordingly, in next experimental tests, Zigbee-based ranging and CMDS will be mostly considered within local portions of road at reasonable speed (typically, at urban intersections, on roundabouts), while optimizing both the number and the relative configuration of static anchors.

\section{CONCLUSION}

We have reviewed and illustrated the main components of a service platform to provide highly accurate localization for C-ITS, developed in the EU H2020 HIGHTS project, where heterogeneous technologies, sensors, algorithms, services and protocols come together to provide accurate localization capable to meet the aforementioned requirements.
We described preliminary off-line validations of HIGHTS algorithms, based on the integrated platform. These first results not only show encouraging gains beyond nominal GPS, but they also provide useful field feedback for the next integration and demonstration steps scheduled in the project.

\section{ACKNOWLEDGMENT}

This work has been supported by the EU project HIGHTS, grant no. 636537. Authors want also to explicitly thank all contributors and leaders of HIGHTS deliverables or related scientific papers, and in particular: A. Ghods, A. Stoica, G. Abreu, I. Khan, G. M. Hoang, D. Slock, N. Garcia, M. Raffero, V. Comito, C. Dannheim, O.-P. Berger, M. Walter, R. Raulefs and C. Götze. EURECOM acknowledges the support of its industrial members, namely, Orange, BMW Group, SAP, Monaco Telecom, Symantec, IABG. Thanks to Objective Software $\mathrm{GmbH}$, Ibeo Automotive Systems $\mathrm{GmbH}$, ZigPos $\mathrm{GmbH}$, BeSpoon and TASS-Siemens for having provided vehicles, hardware and facilities.

\section{REFERENCES}

[1] ETSI, ETSI TR 102863 v1.1.1 (2011-06), Intelligent Transport Systems (ITS); Vehicular Communications; Basic Set of Applications; Local Dynamic Map (LDM); Rationale for guidance on standardization, 2011.

[2] ETSI, ETSI EN 302895 v1.0.0 (2014-01), Intelligent Transport Systems (ITS); Vehicular Communications; Basic Set of Applications; Local Dynamic Map (LDM), 2014.

[3] G. Soatti, M. Nicoli, N. Garcia, B. Denis, R. Raulefs, and H. Wymeersch, Implicit Cooperative Positioning in Vehicular Networks," IEEE Trans. on Intelligent Transportation Systems (to appear), 2018.

[4] M. Fröhle, C. Lindberg, H. Wymeersch , Cooperative Localization of Vehicles without Inter-vehicle Measurements, IEEE Wireless Communications and Networking Conference (WCNC), 2018.

[5] G. M. Hoang and B. Denis and J. Härri and D. T. M. Slock, Cooperative localization in GNSS-aided VANETs with accurate IR$U W B$ range measurements, 13th Workshop on Positioning, Navigation and Communications (WPNC) 2016.

[6] HIGHTS Project, D5.2 - Specifications of implemented cooperative and fusion algorithms [intermediary], www.hights.eu [Online], 2017.

[7] HIGHTS Project, D3.3 - Protocols \& Facilities (Cooperative Communications) [Final], www.hights.eu [Online], 2017.

[8] C. Gentner, T. Jost, W. Wang, S. Zhang, A. Dammann, and U.-C. Fiebig, Multipath Assisted Positioning with Simultaneous Localization and Mapping, IEEE Trans. Wireless Commun., vol. 15, no. 9, pp. 6104 6117, Sep. 2016.

[9] M. Ulmschneider, C. Gentner, T. Jost, and A. Dammann, Multiple Hypothesis Data Association for Multipath-Assisted Positioning, 14th Workshop on Positioning, Navigation and Communications (WPNC) 2017.

[10] A. Ghods, G. Abreu and S. Severi, Cholesky MDS: A fast and efficient heterogeneous localization algorithm, 2017 IEEE International Conference on Communications Workshops (ICC Workshops), pp. 1055-1060, Paris, 2017.

[11] G. M. Hoang and B. Denis and J. Härri and D. T. M. Slock, Mitigating unbalanced GDoP effects in range-based vehicular Cooperative Localization, IEEE International Conference on Communications Workshops, 2017.

[12] G. M. Hoang and B. Denis and J. Härri and D. T. M. Slock, Breaking the Gridlock of Spatial Correlations in GPS-Aided IEEE 802.11pBased Cooperative Positioning, IEEE Trans. on Vehicular Technology, 2016.

[13] G. M. Hoang and B. Denis and J. Härri and D. T. M. Slock, On communication aspects of particle-based cooperative positioning in GPS-aided VANETs, IEEE Intelligent Vehicles Symposium (IV), 2016.

[14] https://vimeo.com/210411008/80fdb55972 